\title{
Gender dimorphism in differential peripheral blood leukocyte counts in mice using cardiac, tail, foot, and saphenous vein puncture methods
}

\author{
Diana C Doeing, Jessica L Borowicz and Elahé T Crockett*
}

Address: Departments of Physiology and Surgery, College of Human Medicine, Michigan State University, East Lansing, MI, USA

Email: Diana C Doeing - doeingdi@pilot.msu.edu; Jessica L Borowicz - borowic3@msu.edu; Elahé T Crockett* - ecrocket@msu.edu

* Corresponding author

This article is available from: http://www.biomedcentral.com/1472-6890/3/3

(c) 2003 Doeing et al; licensee BioMed Central Ltd. This is an Open Access article: verbatim copying and redistribution of this article are permitted in all media for any purpose, provided this notice is preserved along with the article's original URL.

\begin{abstract}
Background: In many animal models that investigate the pathology of various diseases, there is a need to monitor leukocyte counts and differentials. However, various researchers use a range of different techniques in male and female laboratory animals to collect such blood variable information. These studies are then compared to one another without consideration of the possibility that different bleeding sites or techniques as well as gender may produce varying results. In light of this, the peripheral blood leukocyte counts and differentials of C57BL/6 male and female mice were determined using four blood-sampling techniques: cardiac, tail, foot, and saphenous vein punctures.
\end{abstract}

Methods: Blood smears were prepared and stained with Wright-stain for differential cell analysis. The total number of peripheral blood leukocytes was determined with the aid of a hemocytometer. Applying ANOVA and Student $t$-test analysis made comparisons between groups.

Results: The total leukocyte counts obtained using the cardiac puncture method were significantly lower as compared to the other three blood sources; saphenous, tail and foot. There were no significant differences between leukocyte counts of blood samples collected from the tail, saphenous, and foot. Additionally, no significant differences were observed in total leukocyte counts between male and female mice. Differential analysis showed lymphocytes as the predominant cell type present in the peripheral blood of both male and female mice, comprising 75-90\% of the total leukocytes. While no significant differences were observed between male and female differential counts of blood collected from saphenous and tail veins, a significant difference in differential counts of blood obtained via cardiac puncture was observed between the male and female groups, suggesting the role of sex hormones. Further, of the four methods, cardiac puncture appeared to be the fastest and more reliable technique, yielding the maximum blood volume with the least amount of stress being exerted on the sampling site.

Conclusions: This information suggests that in studies concerning leukocyte counts and differentials, the animal gender and the sampling site of blood collection should be kept consistent as to avoid introducing any misleading experimental variation, and that cardiac puncture is the best method of blood collection in mice. 


\section{Background}

Many studies that explore the pathology of various diseases plaguing both veterinary and human medicine utilize animal models. In using these models, leukocyte count and differential information is often monitored, since this data can be a useful marker in evaluating the effectiveness or outcome of certain treatments. However, the source of such blood variable information is often not consistent. Investigators utilize different techniques and genders within the same study. For example, blood may be collected from a puncture in the animal's tail vein throughout the duration of the study, but if the chest cavity is open at the end of the study to collect other tissue samples, the final blood sample is collected from the heart. This practice is not uncommon and can be found in many types of studies using animal models. When different techniques are used in a single study, though, it introduces a means for error. Previous studies in rats have shown significant differences in plasma and blood variables when different methods of bleeding were applied [1$3]$. For example, significant differences between the levels of some hematological parameters (i.e., pCO2, pO2, pH, and $\mathrm{K}+$ ) in blood samples collected by orbital venous plexus and saphenous punctures have been reported [3]. In contrast, in a study using blood samples from the orbital plexus and the venous sublingualis, no significant differences were observed in the serum chemistry values in rats [1]. Further, significant differences between the concentrations of leukocytes in blood samples collected from the heart and eye have been reported in rodents [48]. These studies clearly suggest that the source of blood sampling is an important factor effecting the hematological and plasma variables outcomes. Further, studies indicate that sex steroid hormones have important regulatory functions in circulating leukocytes [9-11]. While the use of mice as laboratory animals has significantly increased no study has examined the effect of blood sampling and gender in mice. Thus, the goal of this study was to investigate different methods of blood drawing with respect to leukocyte counts and differential data in male and female mice. To accomplish this, blood samples were collected from $\mathrm{C} 57 \mathrm{BL} / 6$ male and female mice using the techniques of cardiac puncture and venous puncture of the tail, foot, and saphenous.

\section{Methods}

\section{Preparation of Animals}

Male and female C57BL/6 mice (Charles River Laboratories, Portage, MI) ranging from ages eight to ten-weeks-old were allowed to acclimate in the animal housing area for about one week before experimentation. During this acclimation period, animals were fed a standard diet and allowed unlimited access to food and water. All mice received humane care in accordance with the Guide for the Care and Use of Laboratory Animals (National Insti- tutes of Health Publication No. 85-23, revised 1985). Aspects of each individual procedure regarding sample size collected, manipulation, and blood flow quality were documented. All the procedures were carried on under standard laboratory conditions at room temperature, $22^{\circ} \mathrm{C}$. Blood samples were collected from four sampling sites, tail, foot, lateral tarsal (saphenous), and heart, in succession from anesthetized mice. The collection of blood samples from all the sampling sites on each animal was carried on in less than seven minutes by assistance of two technicians, and the blood samples were processed for analysis immediately after blood was collected.

\section{Anesthesia}

All animals were lightly anesthetized with inhaled methoxyflurane (Baxter Caribe, Inc., Guayama, PR) to simply immobilize the animal. In this study because blood was drawn in succession from four different sampling sites, and since the cardiac puncture should always be carried out under general anesthesia, all animals were under anesthesia. However, blood can be collected from tail, foot, or saphenous vein of an un-anesthetized mouse by restraining the animal. The selection of this anesthesia was based on its routine application in our laboratory and extensive previous application in many published research reports. Although current application of this anesthesia is limited in the Unite States, it is widely used in many other countries.

\section{Tail Vein Draw}

The anesthetized mouse was positioned in a ventral position so that a dorsal lateral vein could be located. The side of the mouse's tail from which the sample was actually drawn varied depending on the quality of vein appearance through the skin. The tail was gently washed using a piece of gauze and warm water in order to clean the skin and enhance blood flow. Once a vein was selected, a thin layer of petroleum jelly was applied to the skin over the vein topically using a cotton swab, which made the vein more visible as well as prevented the dispersion of the blood over the skin. A 25-gauge hypodermic needle (Becton Dickinson and Co., Franklin Lakes, NJ) was used to make the puncture, at approximately $1 / 3$ of the length of the tail from the tail base. The needle was immediately removed and $40 \mathrm{ul}$ of blood was collected using a Microvette ${ }^{\varpi}$ collection capillary tube lined with heparin (model CB 300LH, Sarstedt Inc., Newton, NC). In some cases, the tail was gently massaged to enhance blood flow when necessary.

\section{Foot Vein Draw}

Following completion of blood drawing from the tail vein, the foot was prepared for a similar procedure. A technique similar to that used for conscious mice was applied by placing the head and trunk in an in-house-made con- 
tainer, composed of a $50 \mathrm{ml}$ plastic centrifuge tube with holes for ventilation, leaving the hind legs free. One leg was extended out and held between the forefinger and third finger of the technician. The dorsum of the foot was rubbed gently in order to locate a quality vein and facilitate blood flow. Petroleum jelly was then spread on the skin over the vein using a cotton swab. The vein was punctured with a 25-gauge hypodermic needle and $40 \mathrm{ul}$ of blood collected with a CB 300LH Microvette ${ }^{\circledast}$ collection capillary tube. The mice were under light anesthesia during the procedure.

\section{Saphenous Vein Draw}

This procedure followed the completion of blood drawing from the foot. The opposite hind leg of the mouse was used. Hair located on the mouse's hind leg over the lateral saphenous vein was shaved using a pet trimmer (Wahl Clipper Corp., Sterling, IL). The lateral saphenous vein was raised by compression of the leg skin with the thumb and forefinger. Once again, petroleum jelly was applied to the skin over the vein and the lateral saphenous vein was punctured using a 25-gauge needle. The needle was removed immediately and $40 \mathrm{ul}$ of blood was collected from the skin's surface with a CB 300LH Microvette ${ }^{\varpi}$ blood collection capillary tube. The mice were under light anesthesia during the procedure.

\section{Cardiac Puncture}

Cardiac puncture was performed following the saphenous vein procedure while the mouse was under anesthesia with inhaled methoxyflurane. The footpad of each animal was pinched a few times to ensure that all physical sensation had been lost. A 3-CC syringe with 25-gauge needle (Becton Dickinson and Co., Franklin Lakes, NJ) was precoated with heparin lock flush solution (100 USP units/ $\mathrm{ml}$, Abbot Laboratories, Chicago, IL). The mouse was placed dorsally and the left chest wall was felt between the thumb and forefinger as to locate the beating heart. The needle of the syringe was lined up laterally above the chest wall in order to estimate how far the needle would need to be inserted into the chest to reach the heart. The needle was then carefully inserted laterally into the chest cavity, and slowly introduced into the cavity until the heart was punctured and blood began to rush into the syringe. The plunger of the needle was then gently aspirated as to collect as much blood from each cardiac cycle as possible and not collapse the heart. Using this technique, approximately 0.7 to $1.0 \mathrm{ml}$ of blood was collected. Following the cardiac puncture the animal was euthanized per protocol approved by the Michigan State University Animal Use and Care Committee.

\section{Leukocyte Count and Differential}

Immediately following blood collection, $5 \mathrm{ul}$ of blood was used to prepare blood smears, which were stained
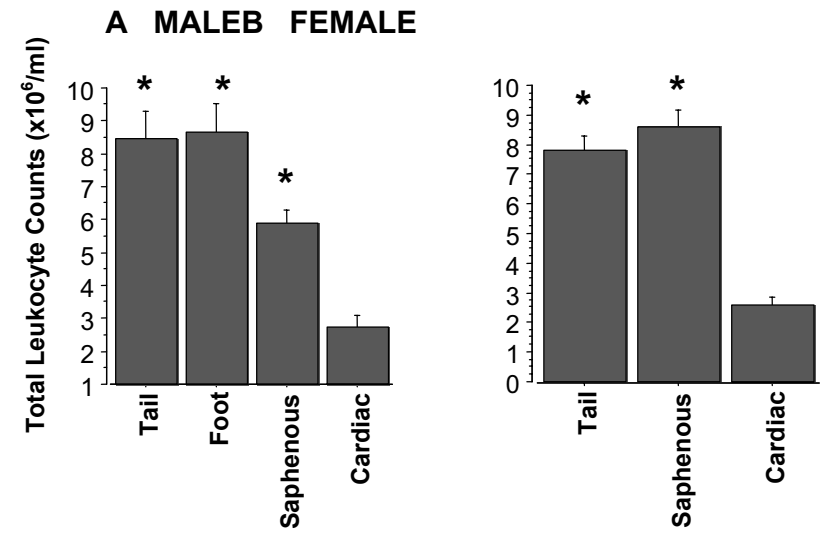

Figure I

Peripheral blood leukocyte counts in male and female mice. Blood samples were collected into heparinized containers from male and female mice via cardiac, tail vein, foot vein, and saphenous vein punctures. Data are expressed as mean \pm SEM $\left(x 10^{6} / \mathrm{ml}\right)$. $* p<0.05$ as compared to cardiac value within the same sex group. The number of mice per each group were; Tail $=14$, Foot $=18$, Cardiac $=17$, and Saphenous $=8$.

with Wright-stain (LeukoStat, Fisher Scientific, Pittsburg, PA) for differential cell counts. For the total leukocyte count, blood was diluted with $3 \%$ acetic acid in saline solution as to lyse all the erythrocytes. Dilutions of 1:100 or 1:20 were prepared, and the Unopette ${ }^{\circledast}$ microcollection system (Becton Dickinson and Co., Franklin Lakes, NJ) was used to prepare the dilutions. All samples were mixed using a Vortex prior to evaluating. Leukocytes were counted using a Neubauer hemocytometer.

\section{Statistics}

All data were expressed as means \pm standard error of the mean. Comparisons between two methods of blood draw were performed using an unpaired t-test by way of StatView version 5.0.1 software ${ }^{\odot}$ for Windows. Comparisons between multiple techniques of collecting blood were also analyzed using a two-way ANOVA with subsequent Student-Newman-Keuls test. $\mathrm{P} \leq 0.05$ was considered significant.

\section{Results and Discussion}

In this study, significant differences were observed between the total peripheral leukocyte counts of blood samples collected via the cardiac puncture and the other three methods applied in mice; tail vein, foot vein, and saphenous vein punctures (Figure 1A). It was consistently observed that leukocyte counts were lowest in blood collected from the heart. The blood samples collected by car- 
Table I: Peripheral blood leukocyte differential counts in male and female mice. Blood samples were collected into heparinized containers from male and female mice via cardiac, tail, and saphenous punctures. Blood smears were prepared and stained with the Wright stain. Leukocyte differential counts were determined by using a $40 x$ objective lens of a light microscope. The monocyte counts were $\mathbf{2} \pm I$ and are not shown on the table. Data expressed as mean \pm SEM. * $p \leq 0.05$ as comparison made between male and female. $n$ represents the number of mice.

\begin{tabular}{lcccc}
\hline $\begin{array}{l}\text { Mouse sex } \\
\text { Blood source }\end{array}$ & Male & Female & Male & Female \\
\% Lymphocytes
\end{tabular}

diac puncture had leukocyte counts of about 2-3 folds less than those obtained by use of the other techniques, i.e., tail, foot, and saphenous. There were no significant differences in total leukocyte counts among the samples taken from the tail, foot, and saphenous veins as compared to each other. In addition, of the four methods applied, cardiac puncture appeared to be the fastest technique, yielding the maximum blood volume with little physical stress being applied to the site of blood sampling. A fairly large volume of blood (i.e., 0.7 to $1 \mathrm{ml}$ ) can easily be collected via a cardiac puncture, while a much smaller volume ( 30 to $100 \mathrm{ul}$ ) is possible to be collect through the tail, foot, or saphenous vein punctures with variable degrees of difficulty. However, an advantage of blood drawing from the foot, saphenous, or tail is that it can be performed on a conscious mouse if application of a light anesthetic is not an option. On the other hand, cardiac puncture requires the use of anesthetics.

Experimental animals with different genders are applied in various studies and gender dimorphism appears to play a role in host response. Thus, it was of interest to examine the effect of sampling sites on the level of blood leukocytes found in female mice. Because there were no significant differences between blood samples collected from the tail, foot and saphenous veins, only two of these techniques, i.e. tail and saphenous, were applied along with cardiac puncture in this part of the study. Similarly, the results indicated that blood collected from the female heart had the lowest leukocyte counts when compared to blood collected from the tail and saphenous veins (Figure 1B). While there were no significant differences between the total leukocyte counts of male and female blood samples, a significant difference in differential leukocyte counts was observed between female and male blood samples obtained by cardiac puncture. The percentage of neutrophils in blood collected via cardiac puncture in female mice was significantly lower than those in male mice (Table 1). Additionally, when the absolute numbers of neutrophils were calculated the difference was statistically significant between the neutrophil counts of blood collected from the hearts of male and female mice (absolute neutrophil counts: female: $0.196 \pm 0.05 \times 10^{6} / \mathrm{ml}$, and male: $\left.0.638 \pm 0.136 \times 10^{6} \mathrm{ml}, p<0.001\right)$. There were no significant differences between blood samples of male and female mice obtained from the tail vein or saphenous vein. The data suggests that sex steroids have an effect on the regulation of circulating leukocytes. It has been shown that female steroid hormones, 17 beta-estradiol and progesterone, can modulate the expression of adhesion molecules on human peripheral blood leukocytes, thereby, affecting their circulation and activation [11]. In women, a consistent fall in neutrophil count at menstruation has been shown [12]. The fall in neutrophil counts may be related to infiltration of neutrophils into the uterus as has been demonstrated in mice treated with estradiol [13]. Further, the extensive experience of our laboratory (unpublished data) of working with human blood leukocytes as well as published studies [14], indicate a great variation in the concentrations and cellular responses of white blood cells of healthy adult female individuals, which correlate to various phases of the menstrual cycle. Other published studies in animals have also indicated the influence of the reproductive state (i.e., estrous cycle) on neutrophil concentrations and functions [10]. It must be noted that in our study presented here, the phases of the estrus cycle were not identified. Further study is required to evaluate the role of sex hormones and cyclic changes on the concentrations of peripheral blood cells. The data presented in this study and the previously published studies collectively suggest the effect of sex steroids on circulating leukocytes, which should be considered in experimental design and interpretation of data.

Despite the significant differences presented between the neutrophil counts of blood samples drawn from the hearts of female and male mice, no significant difference was present when blood samples were drawn from tail and saphenous veins. One potential explanation may be due to the influence of the sampling techniques. In blood samples taken from the tail and saphenous, local physical stress may have caused the release of marginating leuko- 
cytes into circulation, falsely changing the leukocyte concentrations of the circulating blood. However, such local manipulation was not introduced during the cardiac puncture. Therefore, the effect of female steroid hormones on circulating leukocytes can easily be appreciated in blood drawn from the heart and not of the samples collected from the tail and saphenous veins. Some investigators have speculated that differences in vessel diameter and flow dynamics account for the leukocyte counts obtained at various sampling sites. No striking variation in vessel diameter was observed between the male and female mice used in our study. Although the existence of flow dynamic variability between male and female mice was not examined in this study, the male and female mice appeared to exhibit similar heart beats. However, the variability in vessel diameter could be a factor contributing to the differences that existed between blood leukocyte counts collected from the heart, tail, foot, and saphenous.

The results of our study of mice are in accord with the results of similar studies involving other rodents $[6-8,15]$. A marked decrease in leukocyte counts has been reported in samples taken from the heart versus the tail of rats $[4,14]$. Additionally, Nemzek et al. have shown significantly lower leukocyte counts in blood collected from the mouse heart when compared to those of the tail and orbital sinus [7]. However, the leukocyte counts of the mice blood in our study (tail: $8.4 \pm 0.9 \times 10^{6} / \mathrm{ml}$, heart: $2.7 \pm 0.5 \times 10^{6} / \mathrm{ml}$ ) were lower than those of Nemzek et al. (tail: $16 \pm 2 \times 10^{6} / \mathrm{ml}$, heart: $4 \pm 1 \times 10^{6} / \mathrm{ml}$ ). The differences may due to the differences in sampling techniques. Nemzek et al. clipped the tail and did a laparotomy to expose the heart for blood sampling. In our study, the tail vein was punctured with a 25-guage needle and blood was collected into a heparin-coated capillary tube. Further, blood was drawn from the heart without the animal being subjected to any surgical procedure for laparotomy. In the Nemzek et al. study, the trauma induced by clipping the tail and the surgical laparotomy might have triggered signals to release the marginated leukocytes into circulation, and thereby reflecting the higher number of leukocyte counts. Another reason may due to the procedure used in blood analysis, in which Nemzek et al used an automated hematology system cell counter while in our study the cell counts were performed manually using a hemocytometer. Furthermore, different anesthetics were used in our study (i.e., methoxyflurane) than those of Nemzek et al. (i.e., ketamine, and xylazine). Studies have shown that the type of anesthetics may have an effect on leukocyte counts $[4,15,16]$. However, the data presented in this study as well as our un-published data from un-anesthetized mice have shown no significant effect of the anesthesia on leukocyte counts of blood collected from the tail, foot and saphenous veins. We have not drawn any blood from the hearts of un-anesthetized mice. Therefore, one must note that the relative counts may change if different anesthetics are applied. Our study supports the previous reports and adds new information regarding blood parameters collected via the saphenous vein and cardiac puncture without opening the animal.

The reason(s) for the differences observed in the leukocyte counts collected from different sampling sites is not clearly understood. One reason may be attributed to the immunological demands of the host at various sites [14]. Another theory is that the vascular resistance and blood stasis created by the larger surface area in the capillary bed may be responsible for the higher leukocyte counts from peripheral sites [5]. Further, it is possible that stressinduced procedures at blood sampling sites may cause changes in the number of leukocytes. For example, there is a great deal of manipulation involved in locating the saphenous, foot, and tail veins for drawing. The areas are rubbed and massaged, while pressure is applied in order to find a good visible vein beneath the skin. On the other hand, this type of manipulation is absent in the cardiac puncture method. In fact, in our experience, the cardiac technique was the easiest of all the techniques to perform. The puncture was made quickly and efficiently, and the blood flowed easily from the beating heart into the syringe. However, further manipulation was often needed after a puncture was made in the other techniques to prevent coagulation and facilitate further flow of blood. Drawing from the saphenous vein was the next best sampling site when compared to the tail and foot veins. This may be attributed to the larger size of the blood vessel, which facilitated a better blood flow with less physical manipulation applied to the site. Additionally, physical manipulation induced by rubbing and/or local warming by exposing the animal to $37^{\circ} \mathrm{C}$ for a few minutes, causes vasodilatation, which promote bleeding [16]. Further, mechanical stimulation induced by rubbing the area causes local release of mediators resulting in the release of leukocytes from the marginal pool into the blood stream, effecting the increase in leukocyte counts. One might argue that the removal of blood via multiple sampling of the tail, foot, and saphenous veins have affected the leukocyte counts of the blood drawn from the heart at the end of the blood sampling. We don't believe this is a significant factor in our studies for the following reasons. We have observed no significant differences between leukocyte counts of blood collected from mice that have only been subjected to cardiac puncture (data not shown in this report) and those that have been subjected to multiple blood draws as shown in this study. Additionally, a study has shown that changing the order of blood sampling did not alter the results of leukocyte counts in mice [7]. Further, studies have shown that removal of blood beyond $15 \%$ can affect some of the hematological parameters such as mean corpuscular volume and red cell counts 
[16]. In our study, a total of $120 \mathrm{ul}$ of blood were drawn via tail, foot, and saphenous vein punctures, which is less than $10 \%$ of total blood volume of the mouse (a mouse of $25 \mathrm{~g}$ body weight has about $1.8 \mathrm{ml}$ blood volume) [16].

In summary, while it is recognized that the cardiac puncture technique is not always practical and cannot be used in ongoing studies, one should at least be aware of the differences in blood variables as a result of sampling technique and gender dimorphisms. Accordingly, investigators should choose the gender and method of blood draw that best fits their needs regarding anesthetic availability, sample volume, and frequency of sample collection, to be used consistently throughout the study. In addition, one should take these differences into account when comparing studies that have employed varying methods of blood collection and genders.

\section{Authors' Contributions}

DD and JB carried out the technical procedures for blood collection, white blood cell analysis and differential counts. DD also assisted in statistical analysis and preparation of the manuscript. EC conceived the study, and participated in its design, coordination, and preparation of the manuscript. All authors read and approved the final manuscript.

\section{Competing Interests}

None declared.

\section{References}

I. Angelov O, Schroer RA, Heft S, James VC and Noble J: A comparison of two methods of bleeding rats: the venous plexus of the eye versus the vena sublingualis. J Appl Toxicol I984, 4:258-60.

2. Dameron GW, Weingand KW, Duderstadt JM, Odioso LW, Dierckman TA, Schwecke W and Baran K: Effect of bleeding site on clinical laboratory testing of rats: orbital venous plexus versus posterior vena cava. Lab Anim Sci 1992, 42:299-30I.

3. Van Herck H, Baumans V, Brandt CJ, Boere HA, Hesp AP, van Lith $H A$, Schurink $M$ and Beynen AC: Blood sampling from the retroorbital plexus, the saphenous vein and the tail vein in rats: comparative effects on selected behavioural and blood variables. Lab Anim 2001, 35:131-9.

4. Quimby FH, Saxon PA and Goff LG: Total white blood cell counts of peripheral and heart blood samples of the rat. Science 1948 , 1 07:447.

5. Quimby FH and Goff LG: Effect of source of blood sample on total white cell count of the rat. Am J Physiol 1952, I 70:196-200.

6. Sakaki K, Tanaka $\mathrm{K}$ and Hirasawa $\mathrm{K}$ : Hematological comparison of the mouse blood taken from the eye and the tail. Exper Anim. 1961, 10:14-9.

7. Nemzek JA, Bolgos GL, Williams BA and Remick DG: Differences in normal values for murine white blood cell counts and other hematological parameters based on sampling site. Inflamm Res 2001, 50:523-7.

8. Smith CN, Neptun DA and Irons RD: Effect of sampling site and collection method on variations in baseline clinical pathology parameters in Fischer-344 rats. II. Clinical hematology. Fundam Appl Toxicol 1986, 7:658-63.

9. Jilma B, Eichler HG, Breiteneder H, Wolzt M, Aringer M, Graninger W, Rohrer C, Veitl M and Wagner OF: Effects of I 7 beta-estradiol on circulating adhesion molecules. J Clin Endocrinol Metab 1994, 79: $1619-24$.

10. Subandrio AL, Sheldon IM and Noakes DE: Peripheral and intrauterine neutrophil function in the cow: the influence of endog- enous and exogenous sex steroid hormones. Theriogenology 2000, 53:|59|-608.

II. Chernyshov VP, Vodianyk MO and Hrekova SP: Effect of female steroid hormones on expression of adhesion molecules by peripheral blood leukocytes. Fiziol Zh 2002, 48:46-53.

12. England JM and Bain BJ: Total and differential leucocyte count. Br J Haematol 1976, 33: I-7.

13. Gaunt SD and Pierce KR: Myelopoiesis and marrow adherent cells in estradiol-treated mice. Vet Pathol 1985, 22:403-8.

14. Northern AL, Rutter SM and Peterson CM: Cyclic changes in the concentrations of peripheral blood immune cells during the normal menstrual cycle. Proceedings of the Society for Experimental Biology and Medicine. 1994, 207:8I-8.

15. Sluiter W, Hulsing-Hesselink E, Elzenga-Claasen I and Van Furth R: Method to select mice in the steady state for biological studies. J Immunol Methods 1985, 76: I35-43.

16. Diehl KH, Hull R, Morton D, Pfister R, Rabemampianina Y, Smith D, Vidal JM and van de Vorstenbosch C: A good practice guide to the administration of substances and removal of blood, including routes and volumes. J Appl Toxicol 200I, 2I: I5-23.

\section{Pre-publication history}

The pre-publication history for this paper can be accessed here:

http://www.biomedcentral.com/1472-6890/3/3/prepub
Publish with Bio Med Central and every scientist can read your work free of charge

"BioMed Central will be the most significant development for disseminating the results of biomedical research in our lifetime. "

Sir Paul Nurse, Cancer Research UK

Your research papers will be:

- available free of charge to the entire biomedical community

- peer reviewed and published immediately upon acceptance

- cited in PubMed and archived on PubMed Central

- yours - you keep the copyright

Submit your manuscript here:

http://www.biomedcentral.com/info/publishing_adv.asp
BioMedcentral 